\title{
Free Flexural Vibration Response of Integrally- Stiffened and/or Stepped-Thickness Composite Plates or Panels
}

\author{
Jaber Javanshir, Touraj Farsadi and Umur Yuceoglu \\ Department of Aerospace Engineering, Middle East Technical University, Ankara, 06531, Turkey
}

(Received 29 September 2012; accepted 21 May 2013)

This study is mainly concerned with a general approach to the theoretical analysis and the solution of the free vibration response of integrally-stiffened and/or stepped-thickness plates or panels with one or more integral plate stiffeners. In general, the Stiffened System is considered to be composed of dissimilar Orthotropic Mindlin Plates with unequal thicknesses. The dynamic governing equations of the individual plate elements of the system and the stress resultant-displacement expressions are combined and algebraically manipulated. These operations lead to the new Governing System of the First Order Ordinary Differential Equations in state vector forms. The new governing system of equations facilitates the direct application of the present method of solution, namely, the Modified Transfer Matrix Method (MTMM) (with Interpolation Polynomials). As shown in the present study, the MTMM is sufficiently general to handle the free vibration response of the stiffened system (with, at least, one or up to three or four Integral Plate Stiffeners). The present analysis and the method of solution are applied to the typical stiffened plate or panel system with two integral plate stiffeners. The mode shapes with their natural frequencies are presented for orthotropic composite cases and for several sets of support conditions. As an additional example, the case of the stiffened plate or panel system with three integral plate stiffeners is also considered and is shown in terms of the mode shapes and their natural frequencies for several sets of the boundary conditions. Also, some parametric studies of the natural frequencies versus the aspect ratio, stiffener thickness ratio, stiffener length (or width) ratio and the bending stiffness ratio are investigated and are graphically presented.

\section{INTRODUCTORY REMARKS AND BRIEF REVIEW}

The so-called integrally-stiffened and/or stepped-thickness plate or panel systems of various configurations are primarily used in air and space flight vehicle structures and substructures. They may also be utilized in high speed hydrodynamic vehicle structural systems. ${ }^{1-3}$ Their applications in engineering are due to their advantageous properties of light-dead-weight, freedom from mechanical (riveted, bolted, or welded) connections, and of their favourable stiffness characteristics in appropriate places. A typical and well-known example is their utilization as aircraft wing cover panels with multi-stiffeners. ${ }^{3}$

These integrally-stiffened plate or panel systems are manufactured by means of the CAD-CAM process as one-piece plate systems out of a solid (or raw stock) of advanced MetalAlloy plates. In some applications, they may also be manufactured as one-piece advanced composite plate systems with some stepped thicknesses. ${ }^{2,3}$

The integrally-stiffened plate or panel systems may generally be categorized or grouped in terms of four main groups as shown in Fig. 1. For the purposes of the present study, the Group I systems may further be organized in terms of the number of steps in their configurations, as shown in Fig. 2. In the present study, the integrally-stiffened plate or panel systems (one-step, two-step, three-step, four-step, etc., and all in one direction) are to be analysed by means of a general approach to their free dynamic response.

Some significant research studies on the aforementioned integrally-stiffened and/or stepped-thickness plates are available in world-wide scientific and engineering literature. ${ }^{4-19}$ In this brief literature survey, one may include the analytic solutions. ${ }^{4,5,10,17,19}$ The Raleigh-Ritz Method, ${ }^{7,9,11,12}$ the Finite Element Method (FEM) ${ }^{18}$ the Finite Strip Method (FSM), ${ }^{13}, 15$ the Kantrovich Method, ${ }^{8}$ and the Superposition Method, ${ }^{16}$ have all been studied. More recently, there appeared some studies by Yuceoglu et al.,20-24 which employed the Modified Transfer Matrix Method (MTMM). ${ }^{20-33}$

The main concern of this study is to present a general approach to the free vibration response of integrally-stiffened and/or stepped-thickness, rectangular Mindlin plates or panels. Thus, the present work aims to achieve two objectives: (1) A general theoretical analysis, and (2) A general method of solution consistent with (1) for the various types of the integrallystiffened plate or panel systems as defined and shown in Fig. 2.

In the present theoretical analysis, as a general approach, the integrally-stiffened and/or stepped-thickness plate or panel system of Group I, Type 4 (or Four-Step case) in Fig. 3 will be considered. Later on, it will be shown that the Lower-Step or Higher-Step cases may easily be obtained from the aforementioned case. Thus, the Group I, Type 4 (or Four-Step case) is presented in terms of its general configuration, material directions, coordinate systems, and the longitudinal cross-section in Fig. 3. Each stiffened plate system is assumed to be a combination of Mindlin plates ${ }^{34}$ with dissimilar orthotropic material properties and unequal thicknesses. In both figures, each plate or panel system is considered to be simply supported at $x=0, a$, while the boundary conditions in the $y$-direction can be arbitrarily specified within the Mindlin plate theory, ${ }^{34}$ which is one of the First Order Shear Deformation Plate Theories (FSDPTs). ${ }^{34,35}$ 